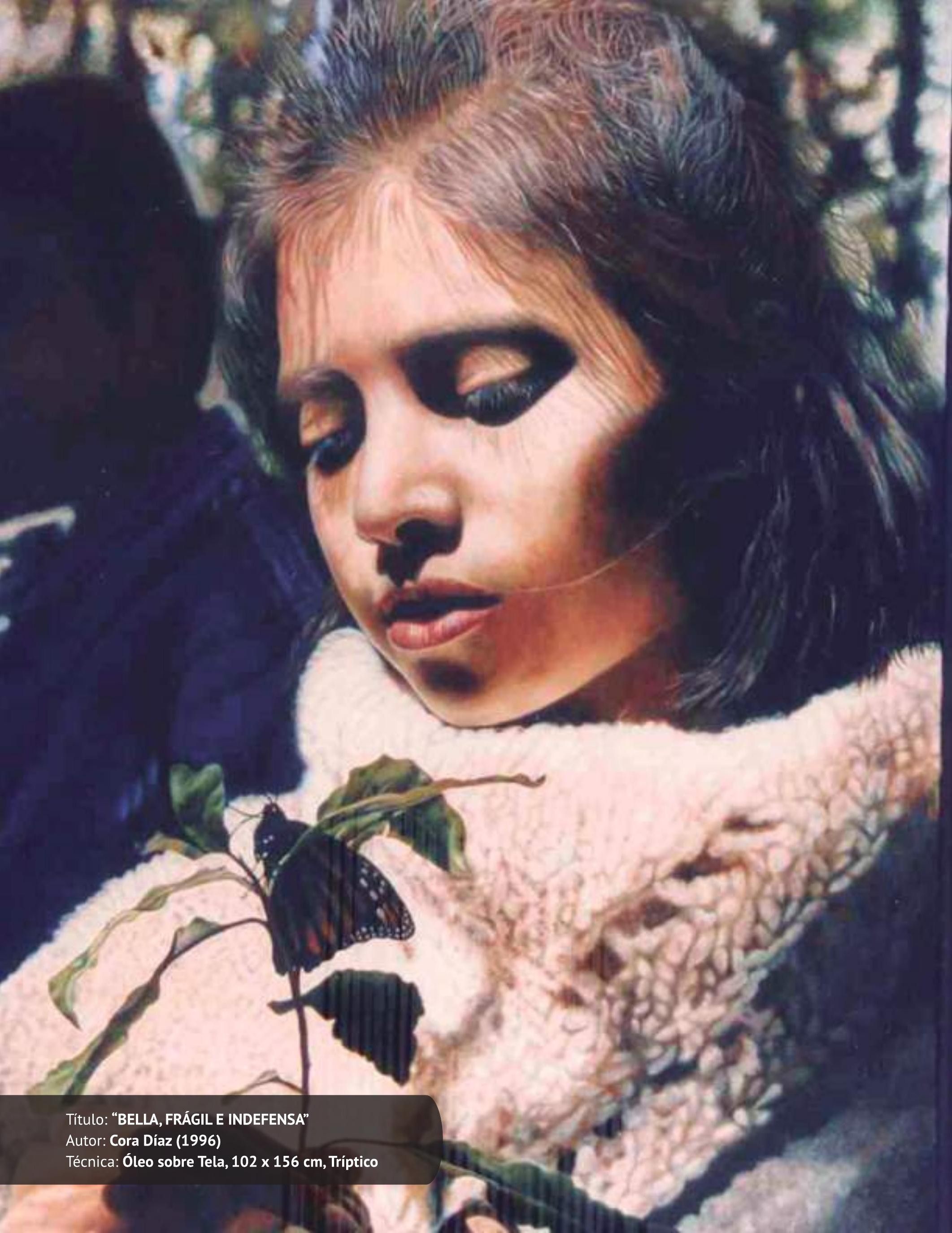




\section{APRENDIZAJE DE UNA LENGUA EXTRANJERA POR ADULTOS A TRAVÉS DEL MÉTODO DE EDUCACIÓN A DISTANCIA}

LEARNING A FOREIGN LANGUAGE BY ADULTS

THROUGH THE DISTANCE EDUCATION METHOD

ROSA MARÍA RÍOS GONZÁLEZ

\section{RESUMEN}

Este proyecto se basa en el análisis de los resultados de aprendizaje de una lengua extranjera en adultos con un método de educación a distancia. El propósito es conocer la influencia de la edad en el aprendizaje de una lengua extranjera en los adultos, las dificultades que estos presentan en el aprendizaje, las ventajas, la importancia de la motivación, el tiempo que se dejó sin estudiar; todo lo anterior es referente al aprendizaje de una lengua extranjera. Uno de los objetivos de este proyecto es encontrar el método apropiado que facilite el aprendizaje del inglés como lengua extranjera.

PALABRAS CLAVE: ADULTOS, APRENDIZAJE, LENGUA, EXTRANJERA, CRÍTICO, DISTANCIA.

\begin{abstract}
This project is based on the analysis of the learning outcomes of a foreign language in adults with a distance education method. The purpose is to know the influence of age in the learning of a foreign language in adulthood, as well as, the difficulties that adult students present in learning, the advantages, the importance of motivation, the time left without studying older adults, all of the above is related to learning a foreign language, one of the objectives of this project, is to find the appropriate method that facilitates learning English as a foreign language.
\end{abstract}

KEYWORDS: ADULTS, LEARNING, LANGUAGE, CRITICAL, FOREIGN CRITICAL DISTANCE. 
E objetivo de esta investigación es identificar los factores tanto favorables como desfavorables en la prueba de evaluación y la prueba final, esto según la edad de los estudiantes. Con la entrevista se intenta identificar el método que facilita el aprendizaje de la lengua extranjera, así como analizar la mejora en los resultados de los estudiantes entre pruebas de diagnóstico y evaluación final, a fin de proponer material didáctico que facilite el aprendizaje del inglés como lengua extranjera de los estudiantes adultos.

Este proyecto basado en la investigación es un análisis de los resultados del aprendizaje de una lengua extranjera en adultos, los cuales estudian en la Modalidad de Educación a Distancia (actualmente llamada Modalidad No Escolarizada y Mixta). Se llevó a cabo en un centro comunitario en Salinas Victoria, Nuevo León, con un grupo de 50 alumnos de entre 15 y 47 años, los cuales cursan la unidad de aprendizaje de Inglés como lengua extranjera.

Con el aprendizaje de una lengua extranjera nos encontramos frente a un panorama dominado por la globalización. Dominar el idioma inglés se ha convertido en una necesidad elemental para enfrentar los desafíos del siglo XXI, así como también la apertura y facilidad de oportunidades de trabajo.

Con respecto al aprendizaje de una lengua extranjera, la UNESCO realiza una declaración mundial sobre la Educación Superior en el siglo XXI (1998). Ésta expresa la necesidad de desarrollar habilidades especializadas como parte de los desafíos que

Constituir un espacio abierto para la educación superior que fomente el aprendizaje permanente, para contribuir a la comprensión, interpretación, promoción y difusión de las culturas nacionales y regionales

$\Delta<\infty<\infty<\infty<\infty<\infty<\infty<\infty<\infty<\infty<\infty<\infty<\infty<\infty<\infty<\infty<\infty<\infty<\infty<\infty<\infty<\infty<\infty<\infty<\infty<\infty<\infty<\infty<\infty<\infty<$

enfrentan las sociedades actuales. En su artículo I (b y d), en referencia a la misión de la Educación Superior, expresa la necesidad de:

Constituir un espacio abierto para la educación superior que fomente el aprendizaje permanente (...) para contribuir a la comprensión, interpretación, promoción y difusión de las culturas nacionales, regionales, internacionales e históricas en un contexto de pluralismo y diversidad cultural.

Así mismo, sobre el aprendizaje de lenguas extranjeras, Ramírez D. (2016) menciona que varios estudios han demostrado recientemente los beneficios cognitivos de aprender un L2 (Segunda Lengua o Lengua Extranjera), lo que ha aumentado el atractivo de esta actividad entre los adultos mayores y puede posicionarlo en un futuro próximo como un potente mecanismo preventivo y terapéutico para disminuir el deterioro cognitivo.

A pesar de esto, este campo aún no ha desarrollado métodos y materiales de enseñanza dirigidos específicamente a este grupo de edad, adaptados a las características cognitivas, psicológicas y sociales del grupo. De hecho, las investigaciones empíricas sobre los alumnos mayores de L2 son limitadas y aún se encuentran en una etapa descriptiva.

La complejidad del aprendizaje de lenguas extranjeras con frecuencia puede ser más difícil para los adultos, esto es debido a la madurez y los cambios cognitivos. Kim (2008) declara que el aprendizaje beneficia a la persona socialmente, ya que promueve la salud y la integración al tiempo que aumenta la calidad de vida. Entre las diversas disciplinas, el aprendizaje de lenguas extranjeras (LE) se lleva a cabo actualmente en un número que ha ido incrementandose entre los adultos mayores.

Por otra parte, y con referencia en la influencia de la edad de los estudiante adultos, Palea y Bostina Bratu (2015) afirman que:

Parece preferible referirse a un rango de factores de la edad en lugar de a un solo factor de edad (p. 431).

Bettoni-Techio M. (2008) y otros autores mencionan que la edad en la adquisición de una segunda lengua ha sido un tema importante en las últimas décadas. En el aprendizaje 


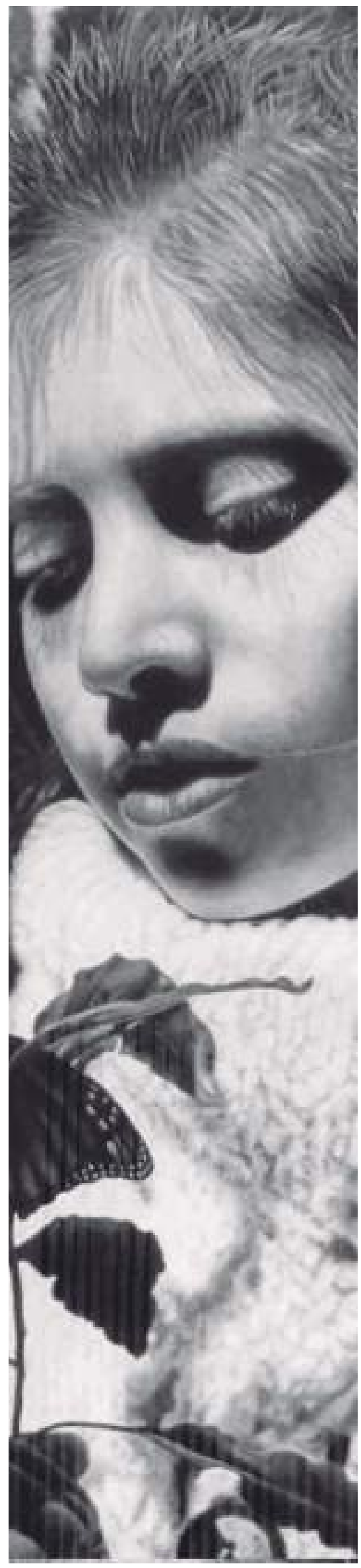

según lo afirmado por Penfiel y Robert (1959), el límite en términos de aprendizaje exitoso del lenguaje es a los 9 años y se debe a limitaciones biológicas como pérdida de plasticidad cerebral.

Algunos investigadores como Gass Selinker (2001) y Nikolov Djigunovic (2006) identificaron algunas desventajas y ventajas en la Hipótesis del Periodo Crítico (HPC) y se descubrió que están relacionadas con los niños y adultos: los adultos son más rápidos que los niños sobre el logro final, pero en competencias los niños alcanzan niveles más altos.

Así como la edad y el aspecto de la motivación,

Los objetivos claros de aprendizaje y la motivación, así como los intereses lingüísticos y culturales específicos, a menudo brindan fuertes motivaciones de aprendizaje a las personas (Dai, N, C, 2012, p.779 en Deng, Zou 2016).

Como resultado, Hubenthal (2004) menciona que, con el tiempo, la motivación y el afecto son factores que influyen en el aprendizaje de una lengua extranjera. Argumentan que la ansiedad afecta el logro en los estudiantes mayores, pero nada se compara con el deseo de aprender una lengua extranjera.

Por el contrario, esto ayuda a desarrollar sus habilidades y confianza. El estudio de Ehrman y Oxford (1995), en el que incluyó algunos sujetos mayores de 60 años, encontró que la confianza en sí mismo y la motivación son muy importantes para respaldar el éxito en el aprendizaje de idiomas extranjeros. Moyer (2004) investigó algunas variables como la motivación, la experiencia en el aprendizaje y la experiencia lingüística, y encontró que la edad de adquisición influye en su logro final.

En este proyecto se pretende mostrar los argumentos de los autores acerca del CPH y su significado. Penfield y Roberts (2014) señalan que el CPH fue introducido por los cirujanos cerebrales; y es más probable que una persona adquiera un idioma si el proceso se inicia antes de los 9 años, ya que el período más oportuno para el aprendizaje de idiomas finaliza antes de la pubertad. Por el contrario, Singleton (2005) determina el Período Crítico como un período limitado, el Djigunovic (2006) identificaron algunas desventajas y ventajas en la Hipótesis del Periodo Crítico (HPC) y se descubrió que están relacionadas con los niños y adultos: los niños alcanzan niveles más altos 


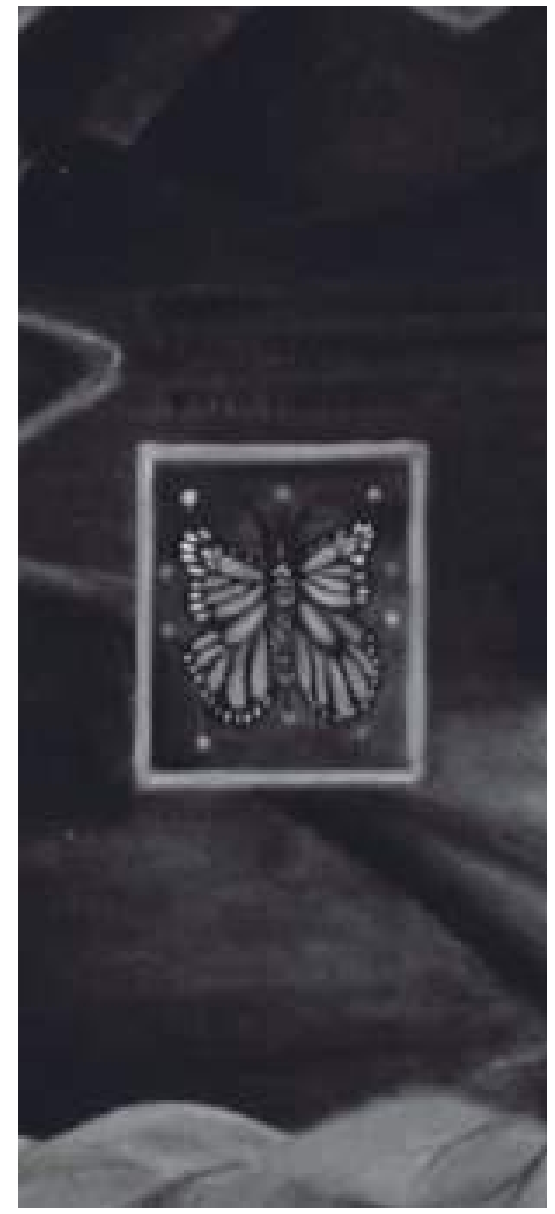

tiempo en que un individuo puede aprender cierta habilidad para incluirla en su comportamiento; Griffiths (2008) incluso menciona la posible existencia de un Período Sensible (SP) en lugar de un Período Crítico (CP). En este caso, dijo que el SLL es mucho más probable después de que este período haya pasado y que el CPH es más determinante.

En este proyecto se trabajó con un grupo de estudiantes de un área rural donde las condiciones de aprendizaje no son las más apropiadas, ya que el aula no tiene las instalaciones adecuadas para su formación y este factor es importante para el aprendizaje de los estudiantes.

La mayoría de ellos son adultos y referente a esto Gardner y Lambert
(1972) mencionan que los antecedentes culturales son una variable que puede volverse potente con la edad de los alumnos. Es importante tener en cuenta que, en una situación de clase, los niños poseen menos conocimientos 0 antecedentes que los adultos, por lo que este problema no parece tener ningún efecto en los jóvenes estudiantes.

Se aplicó una prueba de evaluación diagnóstica validada y diseñada por la Dirección de Educación a Distancia. A través de esta prueba diagnóstica, se identificaron las dificultades de conocimiento previo de los alumnos. La prueba estaba compuesta por 7 ítems; después se aplicó una encuesta diseñada por Antje Carlson, la cual fue adaptada para este grupo de estudiantes a fin de conocer los factores que influyen en el aprendizaje de una lengua extranjera.

En la etapa final se aplicó una prueba piloto para evaluar el avance obtenido al término del curso, ésta contiene 25 ítems y se diseñó de manera congruente con el programa de la unidad de aprendizaje.

La población está compuesta por un grupo de 50 estudiantes de preparatoria en un Centro Comunitario en Salinas Victoria, Nuevo León, de los cuales: 34 son mujeres (68\%) y 16 son hombres (32\%). La edad de dichos alumnos oscila entre los 15 y los 47 años. El rango de 15-20 años son el 56\%, 21-25 años el 24\%, 2630 años el $4 \%, 31-35$ años el $4 \%, 36$ 40 años el 2\%, $41-45$ años el $8 \%$ y $46-50$ años el $2 \%$.

La calificación promedio de la evaluación diagnóstica mostró que la más baja fue de 0.8 y la más alta fue de 100. La mayoría de los resultados de calificaciones son menores a 60 . Entre los estudiantes que fueron evaluados, los rangos fueron de 0-10 (1), $11-30(0), 31-40$ (6), $41-50$ (5), 51-60 (9), 61-70 (18), 71-80 (5), 81-90 (4) y 91-100 (2). La mayoría de los estudiantes califica entre 61 y 70.

El rango de calificaciones de la evaluación final mostró que la calificación más baja fue de 56 , la más alta fue de 100 y la mayor parte es superior a 71. Entre los 50 estudiantes que se evaluaron, el rango de calificaciones fue de 0-50 (0), 51-60 (7), 61-70 (6), 71-80 (16), 81-90 (10) y 91-100 (11). La mayoría de los estudiantes califica entre el rango de 71 y 80. La Tabla 1 muestra los rangos de edad de los estudiantes implicados,

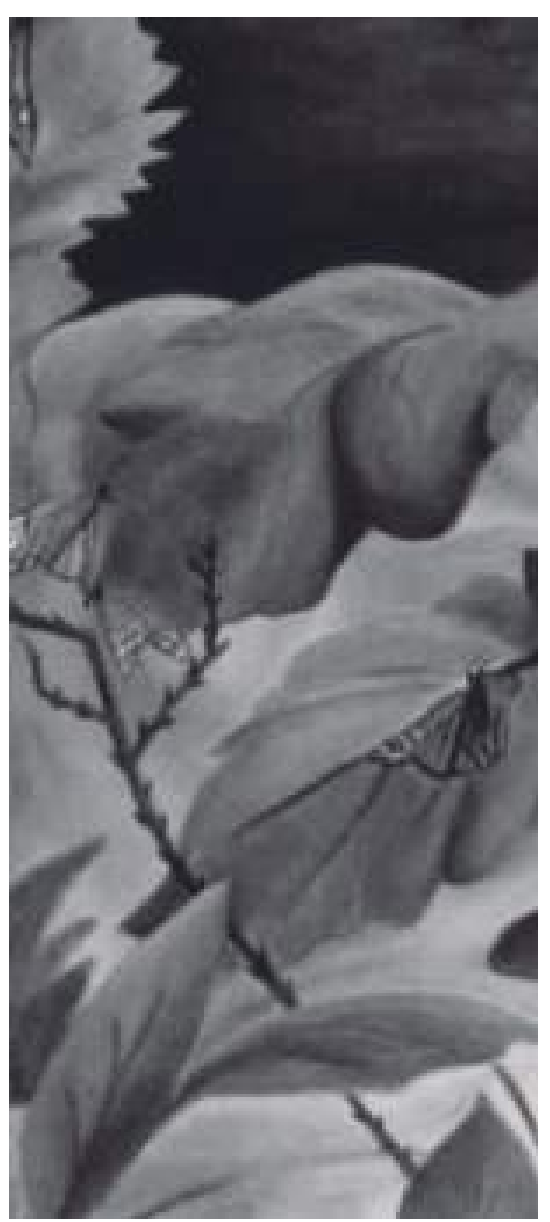


TABLA 1. Resultados de la Evaluación Diagnóstica y Final

\begin{tabular}{|c|c|c|c|}
\hline $\begin{array}{c}\text { RANGO } \\
\text { DE } \\
\text { EDAD }\end{array}$ & $\begin{array}{c}\text { NÚMERO } \\
\text { DE } \\
\text { ESTUDIANTES }\end{array}$ & $\begin{array}{c}\text { CALIFICACIÓN } \\
\text { DE LA EVALUACIÓN } \\
\text { DIAGNÓSTICA }\end{array}$ & $\begin{array}{c}\text { CALIFICACIÓN } \\
\text { DE LA EVALUACIÓN } \\
\text { FINAL }\end{array}$ \\
\hline $15-20$ años & 28 & 63.56 & 78.86 \\
\hline $21-25$ años & 12 & 53.42 & 74.67 \\
\hline $26-30$ años & 2 & 50 & 70 \\
\hline $31-35$ años & 2 & 62 & 82 \\
\hline $36-40$ años & 1 & 62 & 85 \\
\hline $41-45$ años & 4 & 54 & 84 \\
\hline $46-50$ años & 1 & 69 & 92 \\
\hline
\end{tabular}

así como los resultados de la evaluación diagnóstica y los resultados de la evaluación final.

La encuesta Experiencias de adultos en el aprendizaje de una lengua extranjera en el aula de una universidad: un estudio heurístico por Antje Carlson (Adaptado) es la parte de la investigación donde podemos encontrar los resultados sobre lo que piensan los estudiantes acerca del aprendizaje de la lengua extranjera. También responden a las preguntas de esta investigación que son la relevancia de aprender un nuevo idioma en la edad adulta, la motivación y las dificultades que enfrentan en el aprendizaje, el tiempo que algunos de los estudiantes han estado sin estudiar desde que terminaron la educación secundaria, la importancia del contexto social para aprender el idioma inglés $y$, finalmente, el proyecto de propuesta didáctica para

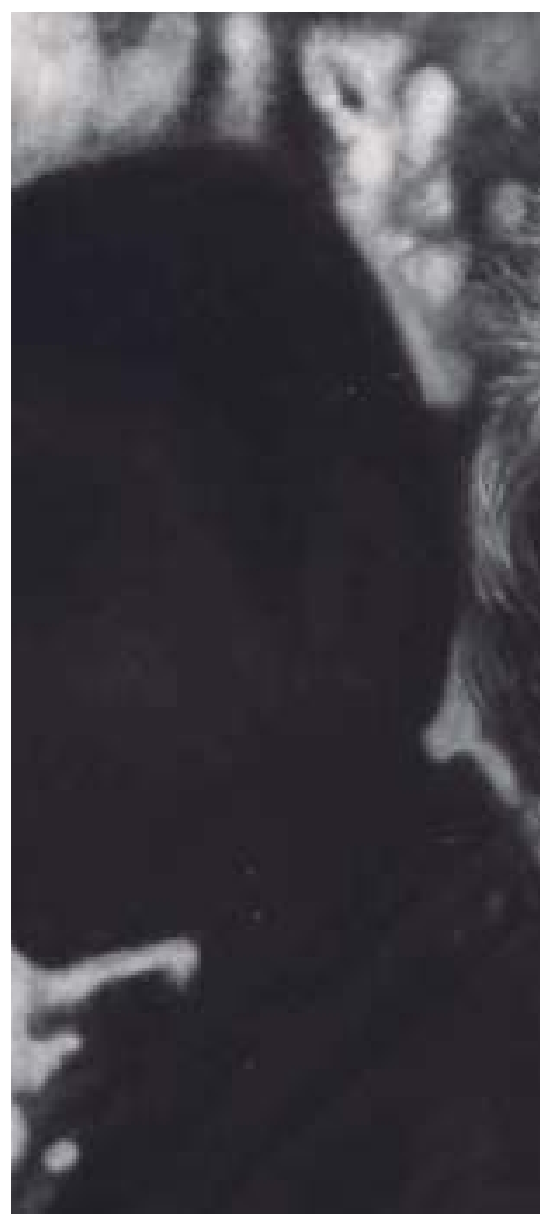

esta investigación, que es el método que facilita su aprendizaje del inglés como lengua extranjera.

En este instrumento se aplicaron 25 preguntas a un grupo de 43 estudiantes adultos. El $51 \%$ respondió que la edad es importante en el aprendizaje de idiomas extranjeros y el $49 \%$ piensan que la edad en el aprendizaje de una lengua extranjera no es importante. Por lo que de acuerdo a los resultados, la edad como factor sí es importante en el aprendizaje de una lengua extranjera.

Los estudiantes mencionan las dificultades enfrentadas en el aprendizaje de una lengua extranjera. El $36 \%$ dijo que la pronunciación es la principal dificultad en el aprendizaje de idiomas extranjeros; un 4\% menciona como factor el tiempo de clase; un $4 \%$ menciona la falta de tiempo, porque no tienen suficiente tiempo 
debido a sus horarios de trabajo; un $8 \%$ menciona la memorización de los vocabularios, un $6 \%$ menciona la habilidad de lectura, un $12 \%$ menciona la habilidad de hablar, un $6 \%$ menciona la habilidad de escritura, un $4 \%$ menciona la falta de dominio del idioma y un $6 \%$ menciona al entendimiento (significado) de algunas palabras como factor en el aprendizaje de lenguas extranjeras.

Según los resultados, el $98 \%$ de los estudiantes menciona que es importante estar motivado para continuar estudiando y el $2 \%$ mencionó que la motivación tal vez puede ser un factor en el aprendizaje de una lengua extranjera.

El porcentaje de tiempo transcurrido sin estudiar de los estudiantes: el $23 \%$ de los estudiantes comenzaron a estudiar inmediatamente al terminar la escuela secundaria, el $18 \%$ comenzaron a estudiar después de 1 año, el $8 \%$ comenzaron después de 2 años, el 10\% después de 3 años, el $5 \%$ después de 4 años, el $10 \%$ después de 7 años, el $3 \%$ después de 8 años, el $3 \%$ después de 9 años, el $5 \%$ después de 10 años, el $3 \%$ después de 16 años, el $3 \%$ después de 19 años, el 3\% después de 20 años, el $3 \%$ después de 26 años, el $3 \%$ después de 27 años, el $3 \%$ después de 28 años y el $3 \%$ después de 32 años de concluir su escuela secundaria. Para los que dejaron de estudiar por más de 3 años les cuesta más trabajo aprender una lengua extranjera.

De los métodos que facilitan el aprendizaje de idiomas extranjeros por adultos: el 39\% mencionó al método de traducción como el mejor para el aprendizaje de una lengua

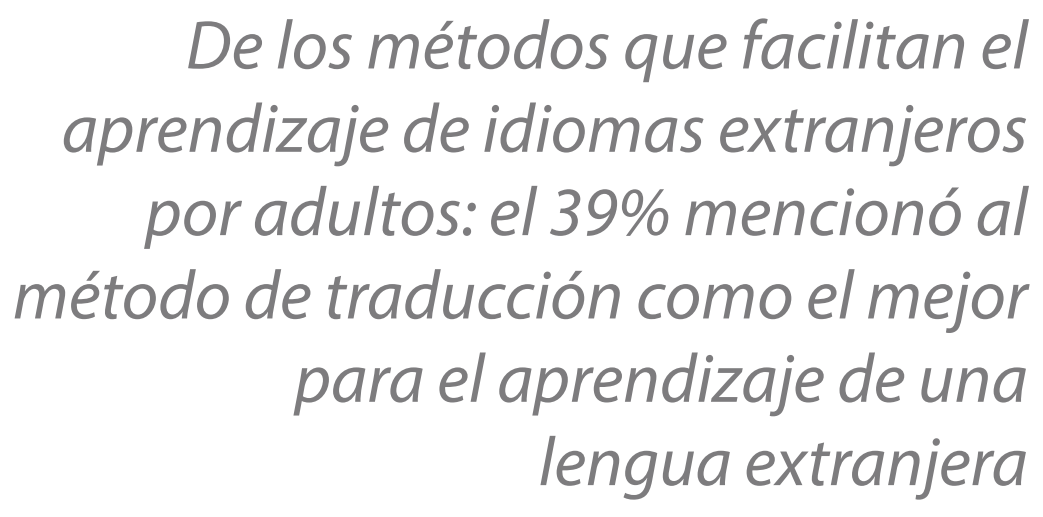

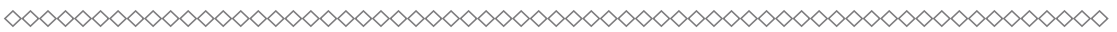

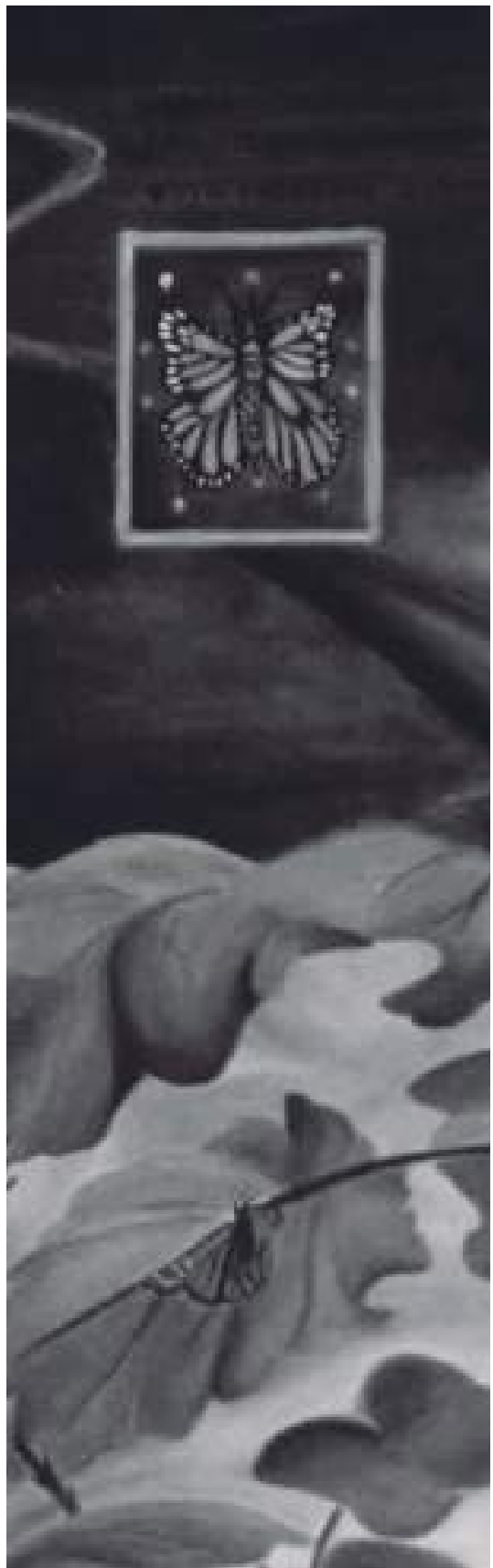

extranjera, el $23 \%$ mencionó que las actividades de pronunciación y lectura les ayudan en el aprendizaje, el $12 \%$ mencionó a las actividades en grupo, el $10 \%$ menciona que el estructurar oraciones a través de la búsqueda del significado de las palabras que no entendieron les ayudan a mejorar su aprendizaje, el 7\% mencionó a las presentaciones de Power Point en las que el docente les dio clase y mostró ayudas visuales, el $5 \%$ mencionó que los videos presentados con la pronunciación de una lista de vocabulario en cada paso de la propuesta didáctica era el mejor método de aprendizaje, el 3\% mencionó el aprendizaje en clase a través de hojas de trabajo y, por último, el $2 \%$ de los estudiantes mencionó que el uso de una aplicación (duolingo) en su teléfono celular era la mejor opción en el aprendizaje de una lengua extranjera.

Para este proyecto fue necesario buscar diferentes autores que ayuden a encontrar la evidencia necesaria que muestre la importancia de un idioma extranjero, las dificultades que enfrentan los estudiantes adultos y el $\mathrm{CPH}$ como un factor importante en el aprendizaje. Y más adelante, a través de los autores, explicar el proceso de 


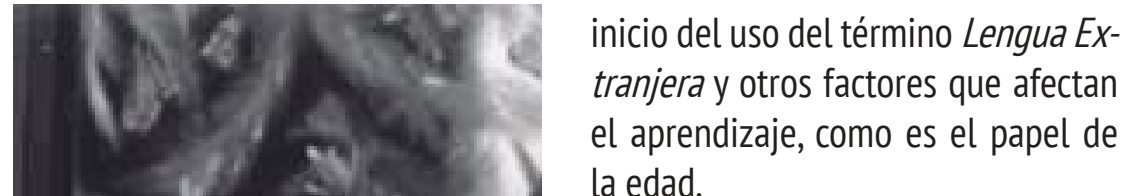
la edad.

De acuerdo con Knowles, Holton y Swanson (1998) se establece que todas las etapas de cambio se adquieren a través de algunos procesos como habilidades, conocimiento 0 experiencia. Brown, Roediger y McDaniel (2014) afirman que el estudiante debe tener en cuenta los conocimientos y habilidades adquiridos para tener oportunidades y dar sentido a problemas posteriores. Según Illich (citado en Harmer, 2007), el aprendizaje no se trata de la actividad de participación en un entorno sin restricciones, sino en un entorno significativo. Entonces, en pocas palabras y resumiendo las creencias de los autores, el aprendizaje es un proceso de instrucción en el cual un ser humano adquiere cierto conocimiento que está listo y presente en su mente.

Como conclusión: Sí es importante la edad en el aprendizaje de una lengua extranjera. También la motivación de salir adelante y encontrar un mejor empleo les ayuda a continuar estudiando. Por otra parte, también se comprueba la Hipótesis del Periodo Crítico en el aprendizaje con el hecho de mencionar que la pronunciación es lo que más complicado les resulta en el aprendizaje de una lengua extranjera. Y, de acuerdo a la Metodología de Investigación Acción, este estudio puede implementarse hasta que la propuesta didáctica arroje los resultados deseados. En cuanto al método aplicado en el proyecto, resultó favorable, ya que los resultados arrogados por las evaluaciones, diagnóstica y final, muestran un incremento positivo en el resultado al momento de compararlos.

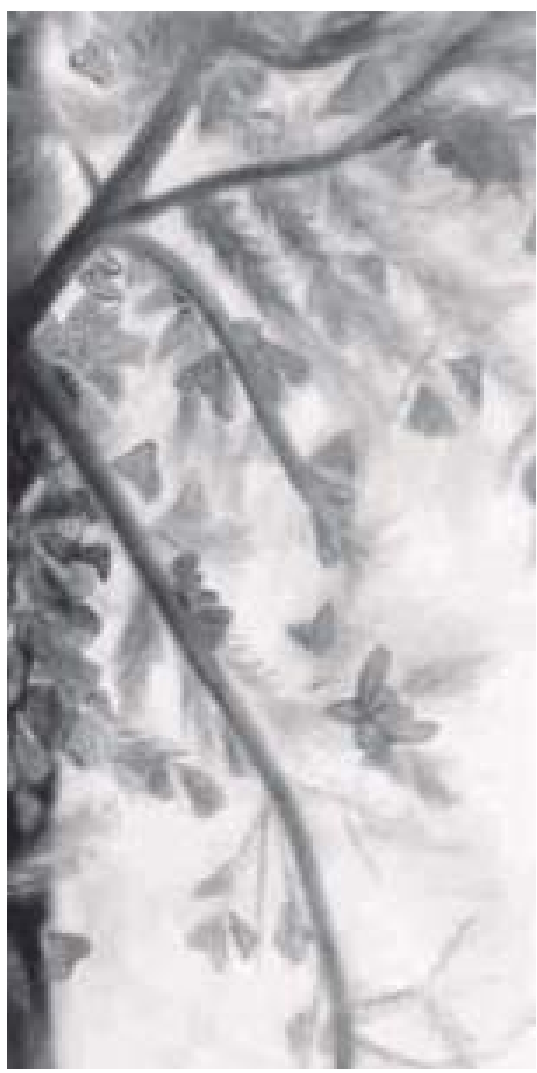

\section{Es importante la edad en el aprendizaje de una lengua extranjera. También la motivación de salir adelante y encontrar un mejor empleo les ayuda a continuar estudiando}




\section{REFERENCIAS}

Bettoni-Techio, M. (2008). Uma discussao atualizada sobre a influencia da idade na aquisicao de L2. Todas as Letras, 10(1), 68-73.

Deng, F., \& Zou, 0. (2016). A study on whether the Adults' second language acquisition is easy or not-From the perspective of children's native language acquisition. Theory and Practice in Language Studies, 6(4), 776-780.

Ehrman, M.E., \& Oxford, R. L.(1995). Cognition plus: Correlates of language learning success. The Moder Language Journal, 79, 67-89.

Gardner, R., \& Lambert, W. (1972). Attitudes and motivation in second language learning. Rowley, Ma.: Newbury House.

Grass, S. M., \& Selinker, L. (2008). Second language acquisition: an introductory course (3rd ed.). Madison Avenue, New York: Routledge.

Harmer, J. (2007). The practice of English Language Teaching (fourth edition ed.). Harlow, United Kingdom: Pearson Logman.

Hubenthal, W. (2004). Older Russian immigrants' experiences in learning English: Motivation, methods, and barriers. Adult Basic Education, 14(2), 104-126.

Moyer, A. (2004). Age, accent and experience in second language acquisition: An integrated approach to Critical Period Inquiry. Clevedon: Multilingual Matters.

Palea, L. L., \& Bostina Bratu, S. (2015). Age and its influence on second language acquisition. Academiei Fortelor Terestre.

Ramírez Gómez, D. (2016). Language teaching and the older adult: Thesignificance of experience. Multilingual matters.

Singleton, D. (2005). The critical period hypotesis: a coat of many colors. International Review of Applied Linguistics(43), 269-285.

UNESCO.(1998). Recuperado el 27 de octubre de 2017 de: http://www.unesco.org/education/ educprog/wche/declaration_spa.htm.

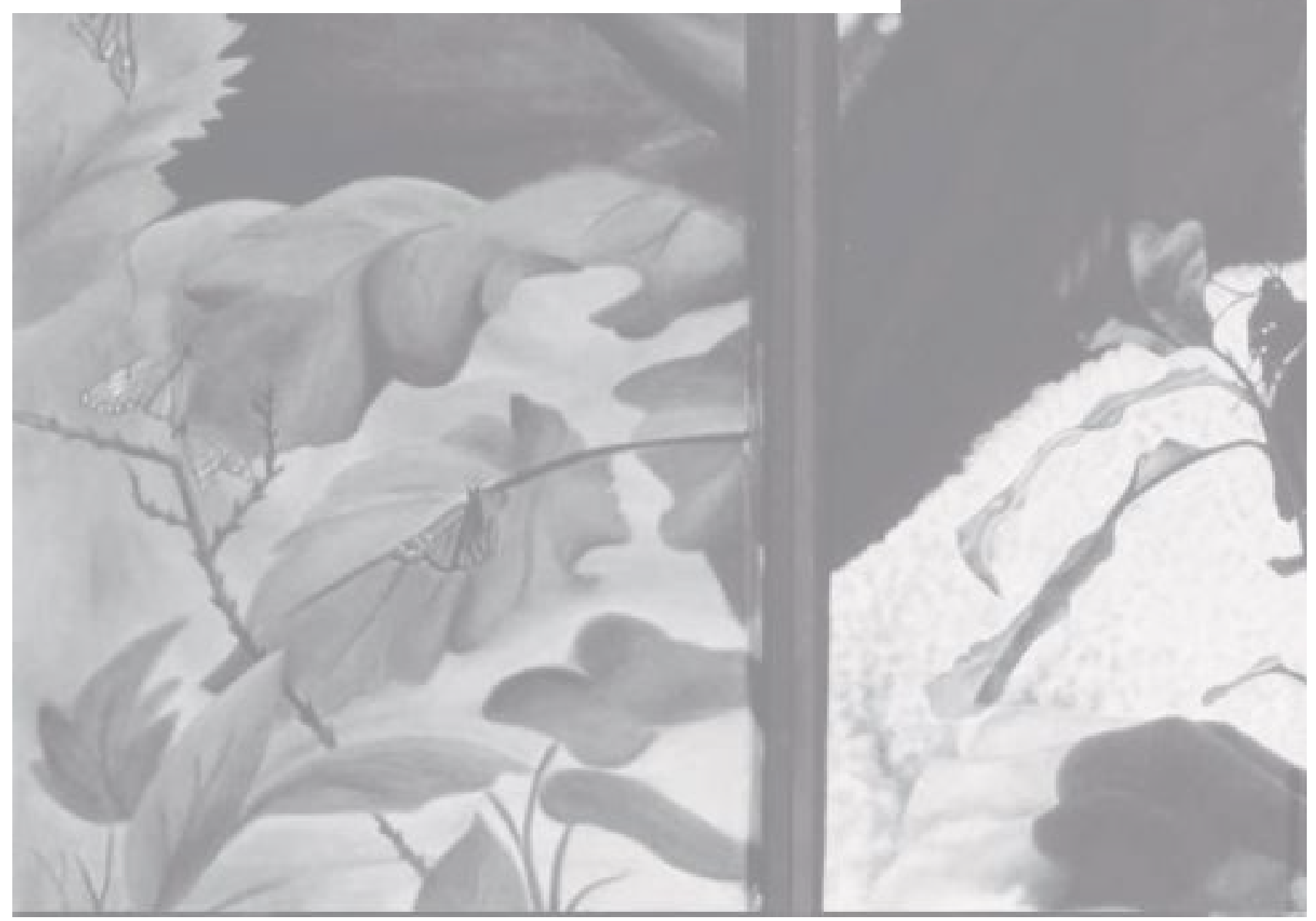




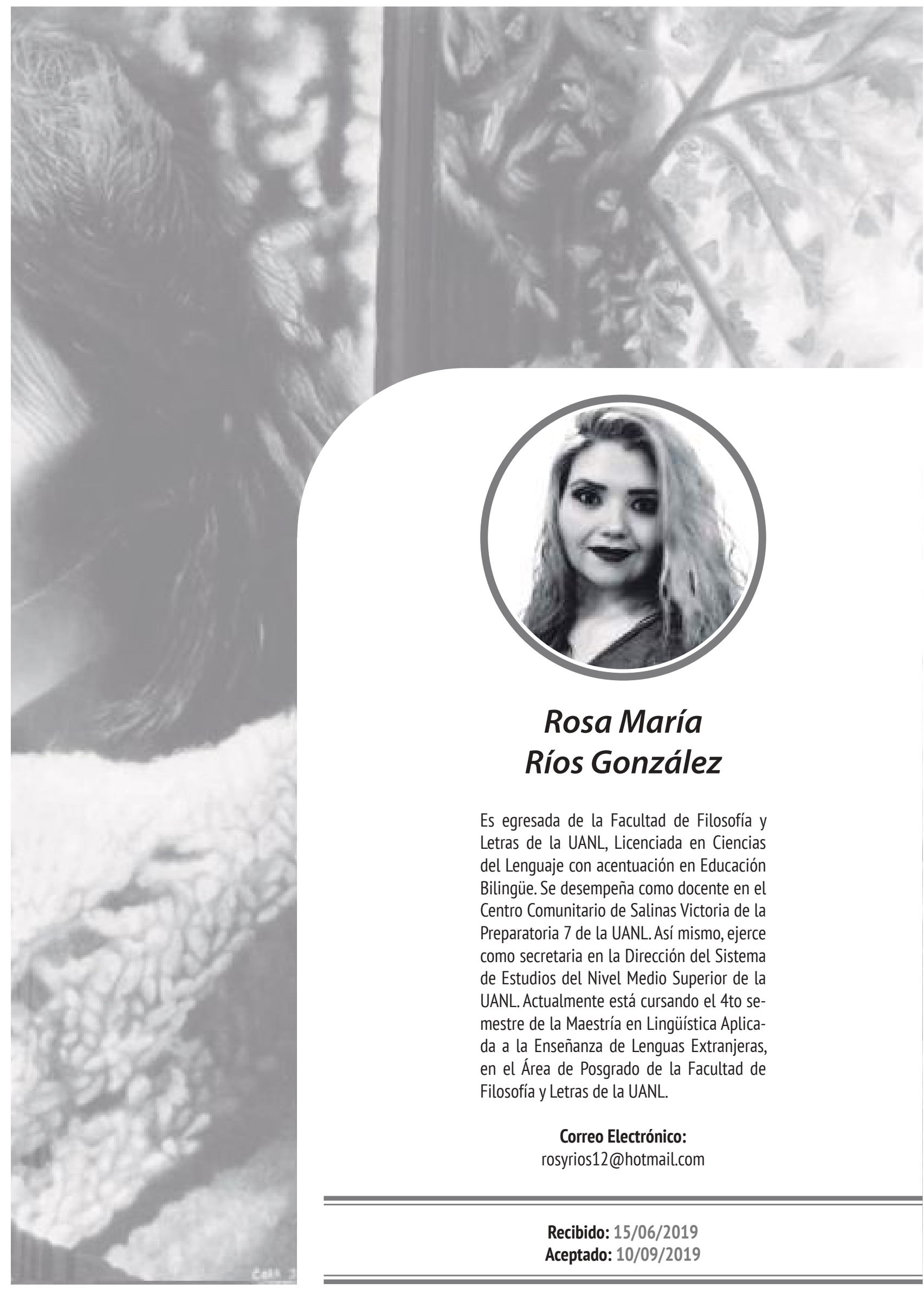

